\title{
A new concrete plastic-damage model with an evolutive dilatancy parameter
}

\author{
Mauro Poliotti, Jesús-Miguel Bairán \\ Department of Civil and Environmental Engineering, Universitat Politcnica de \\ Catalunya - Barcelona TECH (UPC), Jordi Girona, 1-3, Campus Nord, Mod. C-1, \\ 08034 Barcelona, Spain
}

\begin{abstract}
Typical plastic-damage models for concrete use a constant dilatancy parameter. On problems sensitive to confinement and shear softening, this parameter needs ad hoc calibration to fit experimental observations. This makes the model not objective for general applications. To overcome this issue, in this paper, a constitutive plastic-damage model with evolutive dilatancy is proposed for concrete. The evolution of dilatancy is made dependent on the plastic-damage and stress states. The proposed evolution law is validated by comparison of numerical simulations with available experimental results. The validation includes: concrete specimens under uniaxial compression measuring the free expansion, passively confined concrete specimens with different confining materials, and reinforced concrete panels under in-plane shear. It is concluded that the model accurately reproduces concrete lateral expansion through different nonlinear states. Proper modeling of concrete nonlinear expansion proves essential for capturing the response in a number of situations: softening under high shear stresses, confinement, and ductility assessment.

Keywords: Concrete plastic-damage model, Dilatancy, Confinement, Shear strength, Lateral expansion, Softening.

\footnotetext{
Email addresses: mauro.poliotti@upc.edu (Mauro Poliotti), jesus.miguel.bairan@upc.edu (Jesús-Miguel Bairán)

1

Poliotti M, Bairan JM, "A new concrete plastic-damage model with an evolutive dilatancy parameter", Engineering Structures, 2019, doi.org/10.1016/j.engstruct.2019.03.086.
}

Please cite this paper as follows:
\end{abstract}




\section{Introduction}

The assessment of existing structures requires simulation methods able to reproduce serviceability and safety conditions in a precise manner. In the case of reinforced concrete structures in seismic zones, material modeling of concrete is crucial for seismic performance assessment.

Within the same earthquake-resistant structure, there are elements under axial loads with high degrees of confinement and other elements with high shear demands. This requires of material models capable of reproducing a wide range of stress states in an objective manner.

Concrete behavior exhibits a wide range of non-linear phenomena: different responses under tension and compression, large differences in the peak strengths, anisotropy induced by cracking, damage due to the development of micro-cracks, irreversible strains, stiffness recovery upon loading reversals, dilatancy, enhancement in strength and ductility under the effect of confinement, rate dependency, among others.

In particular, dilatancy can be described as the volume change of a granular material when it is submitted to shear strains. This phenomenon plays an important role in the shear behavior of concrete as well as in the increase of strength and ductility due to confinement. This is why its proper representation becomes of especial interest in the evaluation of earthquake-resistant structures.

A large variety of constitutive models for concrete is available on literature with different degrees of approximation and complexity. Most of them are formulated using one or a combination of approaches, such as elasticity, plasticity, damage, and fracture mechanics. Models with coupled plasticity and damage have shown to be able of reproducing the main characteristic of concrete behavior in a robust manner. However, they require especial calibration in those cases where dilatancy is important. For instance, the estimation of the shear strength in reinforced elements, and the simulation of passively confined elements.

This research focuses on the study of plastic-damage models for concrete, especially on the treatment of the dilatancy phenomenon. The development of a plastic-damage model with variable dilatancy for the objective simulation of elements in shear and under confinement is pursued in this paper.

In the following, first a review of the mains aspects of the classical plasticdamage model is made. Further, simulations and comparison with experi-

mental data available in literature are carried out with different values of the 
dilatancy parameter. A novel plastic-damage model with variable dilatancy is proposed to improve the capabilities of the model. The validation of the proposed modifications is made by means of numerical simulation of experimental tests. Three experimental campaigns available in the literature which showed to be significantly affected by concrete dilatancy are reproduced. Finally, conclusions are drawn.

\section{Plastic-damage model with constant dilatancy for concrete}

\subsection{Literature review}

A coupled plastic-damage model for concrete was firstly introduced by Lubliner et al. [1]. There, the classical hardening variable of plasticity theory was replaced by a plastic-damage variable. This was defined as a measure of the energy dissipated during the inelastic process. In a tensile case the dissipated energy is the fracture energy, and in a compressive case is known as the crushing energy, see [2]. Both energies are normalized to avoid meshsensitivity by means of a localization length, see [3; 4]. The model introduced a new yield function and a stiffness degradation variable. The elastoplastic response and the stiffness degradation process were presented in a coupled manner. The single plastic-damage variable enabled the model to reproduce monotonic loading conditions.

Different variants of that model have been developed [5-12]. Furthermore, it has been applied to the simulation of reinforced concrete elements [13-16] showing its capabilities.

In particular, Lee and Fenves [5, 6] presented a modified version of the plastic-damage model in order to include cyclic loading. Two plastic-damage variables, one for tension $\kappa_{t}$ and other for compression $\kappa_{c}$, were introduced. An isotropic stiffness degradation variable was also proposed. In this model, the elastoplastic and stiffness degradation responses (damage) were decoupled. In addition, the control of the tensile and compressive strengths was made by means of the plastic-damage variables. A non-associative flow rule was proposed to control the dilatancy by means of a linear Drucker-Prager function.

This widespread model proved to be able to capture many of the main aspects of the concrete cyclic response. It reproduces plastic deformations, stiffness degradation, crack opening and closing, and different strengths in the tensile and compressive case. It has been implemented in commercial codes, such as ABAQUS [9]. However, the model controls dilatancy with a 
single parameter $\alpha_{p}$ which is held constant. A wide range of values for this parameter was used in literature.

In [5], the effect of the $\alpha_{p}$ parameter in the free expansion of concrete was shown in a biaxial compressive test. It was shown that small values of $\alpha_{p}$ produced significantly smaller values of the out-of-plane strain. This implied that this parameter might need specific calibration for different applications.

One example where this was evidenced is in the research of Genikomsou and Polak [14]. There, the model was applied to the simulation of punching shear in reinforced concrete slabs. In order to fit the experimental data, a parametric study with different values of the dilatancy parameter was carried out. A strong influence in the shear strength and ductility was reported in that research.

Saritas and Filippou [13] simulated the effect of confinement in concrete specimens using the plastic-damage model. Simulations of reinforced concrete beams under both shear and bending were made in the same work. They reported difficulties in the assessment of the strength increase and the post-peak behavior of passively confined elements. To overcome this issue, calibration of the compressive fracture energy was made in that work.

In a different research, Earij et al. [15] performed a parametric study of the dilatancy parameter in the simulation of reinforced concrete beams. A significant loss of ductility for lower values of $\alpha_{p}$ was shown.

Nzabonimpa et al. [16] presented the simulation of concrete beam-column joints using the plastic-damage model. They reported that low values of the dilatancy parameter were not able to fit experimental results.

The need to overcome the previous reported difficulties with a consistent physical approach motivates a closer study of the dilatancy phenomenon. This is the main objective of this research.

\subsection{Basic features}

The cyclic version of the plastic-damage model introduced by Lee and Fenves [5] can be summarized by the following set of equations, see Eqs.(1$3)$,

$$
\begin{gathered}
\overline{\boldsymbol{\sigma}}=\boldsymbol{E}_{0}:\left(\boldsymbol{\varepsilon}-\boldsymbol{\varepsilon}^{p}\right) ; \boldsymbol{\sigma}=[1-D(\boldsymbol{\kappa}, \overline{\boldsymbol{\sigma}})] \overline{\boldsymbol{\sigma}} \\
\dot{\varepsilon}^{p}=\dot{\lambda} \frac{\partial \Phi}{\partial \overline{\boldsymbol{\sigma}}}(\overline{\boldsymbol{\sigma}}) ; \dot{\boldsymbol{\kappa}}=\dot{\lambda} \boldsymbol{H}(\overline{\boldsymbol{\sigma}}, \boldsymbol{\kappa})
\end{gathered}
$$




$$
\dot{\lambda} \geq 0 ; \dot{\lambda} F(\overline{\boldsymbol{\sigma}}, \boldsymbol{\kappa})=0 ; F(\overline{\boldsymbol{\sigma}}, \boldsymbol{\kappa}) \leq 0
$$

where $\boldsymbol{\sigma}$ and $\overline{\boldsymbol{\sigma}}$ are the apparent and effective stresses, respectively, as defined in continuum damage mechanics theory, see [17], among others. $\boldsymbol{E}_{0}$ is the undegraded elastic rank four tensor. $\varepsilon$ and $\varepsilon^{p}$ are the total and plastic strains, respectively. $D$ is the scalar damage variable. The plastic-damage variables, $\boldsymbol{\kappa}=\left[\kappa_{t} ; \kappa_{c}\right]$, play the role of hardening variables. They are defined as the relative measure of energy dissipated during the plastic process. The evolution of plastic strains is defined by means of a non-associative flow rule. $\lambda$ is the plastic consistency parameter, and $\Phi(\overline{\boldsymbol{\sigma}})$ is the plastic potential function. $\boldsymbol{H}(\overline{\boldsymbol{\sigma}}, \boldsymbol{\kappa})$ defines the evolution of the plastic-damage variable. Finally, $F(\overline{\boldsymbol{\sigma}}, \boldsymbol{\kappa})$ is the yield surface that constitutes the threshold of elasticity.

The model is defined by two surfaces, the yield and plastic potential functions. The yield surface presented in Lee and Fenves [5] was an extension to the cyclic case of the yield function known as the Barcelona Model [1]. It can be written under the following form in the effective stress space, see Eq.(4).

$$
\begin{aligned}
F(\overline{\boldsymbol{\sigma}}, \boldsymbol{\kappa})= & \frac{1}{1-\alpha}\left[\alpha \bar{I}_{1}+\sqrt{3 \bar{J}_{2}}\right. \\
& \left.+\beta(\boldsymbol{\kappa})\left\langle\hat{\bar{\sigma}}_{\max }\right\rangle-\gamma\left\langle-\hat{\bar{\sigma}}_{\max }\right\rangle\right]-c_{c}(\boldsymbol{\kappa}) \leq 0
\end{aligned}
$$

where $\bar{I}_{1}=\operatorname{tr}(\overline{\boldsymbol{\sigma}}), \bar{J}_{2}=(\overline{\boldsymbol{s}}: \overline{\boldsymbol{s}}) / 2, \overline{\boldsymbol{s}}$ is the deviatoric effective stress, $\hat{\bar{\sigma}}_{\text {max }}$ is the algebraically maximum effective stress, and $\langle x\rangle=(x+|x|) / 2$ is the Macaulay bracket function. The dimensionless constants, $\alpha$ and $\gamma$, are defined to control the yield surface shape. The $\beta$ function adjusts the relation between the uniaxial strength in tension and compression. The $c_{c}$ function represents the cohesion in compression, and depends on the plastic-damage variable $\boldsymbol{\kappa}$.

The plastic potential function used in Lee and Fenves [5] was the linear version of the Drucker-Prager surface in the effective stress space. See Eq.(5).

$$
\Phi(\overline{\boldsymbol{\sigma}})=\sqrt{2 \bar{J}_{2}}+\alpha_{p} \bar{I}_{1}
$$

where $\alpha_{p}$ is the parameter that controls the dilatant behavior. The linear version of the Drucker-Prager function exhibits singularities in the triaxial isotropic tension point. Therefore, the version implemented in ABAQUS [9] and Omidi and Lotfi [18] used hyperbolic versions of the Drucker-Prager model to overcome this issue. See Eq.(6). 


$$
\Phi(\overline{\boldsymbol{\sigma}})=\sqrt{\left(\varepsilon_{1} \alpha_{p} f_{t 0}\right)^{2}+2 \bar{J}_{2}}+\alpha_{p} \bar{I}_{1}
$$

where $\varepsilon_{1}$ is the eccentricity parameter and $f_{t 0}$ is the uniaxial tensile strength.

The numerical integration of the model was presented in [6], based on the use of the backward-Euler integration scheme in conjunction with a spectral return-mapping algorithm.

\subsection{Dilatancy control}

The dilatancy of the plastic-damage model is controlled through the plastic potential function. A single and constant parameter $\alpha_{p}$ is used for this aim. It can be calculated as follows:

$$
\alpha_{p}=\tan (\psi)
$$

being $\psi$ the dilatancy angle. A constant $\alpha_{p}$ implies a constant dilatancy angle.

Different values of the dilatancy parameter have been proposed in the literature. The tests performed by Lee and Fenves [5] required a value of $\alpha_{p}=0.2$. Similarly, Oller et al. [19] recommended a maximum $\psi=13^{\circ}$ which is equivalent to $\alpha_{p}=0.23$, hence it was consistent with the value used in $[5]$.

Later Genikomsou and Polak [14], in their applications to model punching shear of reinforced concrete slabs, used a dilatancy angle of $\psi=40^{\circ}$ which is equivalent to $\alpha_{p}=0.84$. This value is much bigger than the recommended in $[5 ; 19]$; however, it was needed to capture the shear failure induced by punching.

Earij et al. [15] applied the plastic-damage model to perform 3D simulations of reinforced concrete beams. A sensitivity analysis on this parameter was performed comparing results for $\psi=20^{\circ}, 30^{\circ}, 40^{\circ}, 50^{\circ}$. The conclusion reported in that research was that low values of $\psi$ produced a loss of ductility, so $\psi=40^{\circ}$ was chosen to fit experimental results.

Nzabonimpa et al. [16], in their simulations of beam-column joints, used a dilatancy angle of $\psi=56^{\circ}$, equivalent to $\alpha_{p}=1.48$, to conform the experimental observation in their specimens.

Saritas and Filippou [13], in the modeling of reinforced concrete beams and concrete specimens laterally reinforced, used a constant value of $\alpha_{p}=0.2$. However, in order to adequate the response to the experimental data, these 
authors used different values of the compressive fracture energy for different amounts of transverse reinforcement. The value of the compressive fracture energy used for the biggest ratio of transverse reinforcement was four times bigger than the compressive fracture energy used in the unconfined concrete.

It can be seen that a wide range of values have been used in previous studies. Values between $\alpha_{p}=0.2\left(\psi=13^{\circ}\right)$ and $\alpha_{p}=1.48\left(\psi=56^{\circ}\right)$ have been used in the literature. The applications where only plain concrete was modeled tend to use lower values of the dilatancy parameter $\left(\alpha_{p}\right)$. While, the cases where interaction with reinforcements was simulated needed greater values to properly fit the experimental results. This is inconsistent with the definition of a material parameter, which should be independent of the type of the load configuration.

\subsection{Parametric analysis}

To investigate the influence of the dilatancy parameter, three different types of tests are simulated and compared against experimental data available in literature. Simulations are carried out using the original plasticdamage model introduced by $[5 ; 6]$, with four different values of the dilatancy parameter: $\alpha_{p}=0.1,0.2,0.4$ and 0.6 .

The first test belongs to an experimental campaign carried out by Osorio et al. [20]. There, a uniaxial monotonic compression tests was done measuring the transverse deformation, see Fig.(1). The second test is a passively confined concrete cylinder with a GFRP jacket under uniaxial monotonic loading tested by Aire [21], see Fig.(2). The last experimental data set is obtained from a reinforced concrete panel subjected to in plane shear tested by Vecchio and Collins [22], see Fig.(3).

By analyzing the numerical results, several remarks can be made. It is noteworthy that each test is best fitted with a different value of the dilatancy parameter. In the first test, low values of $\alpha_{p}$ underestimate the post-peak transverse strains, while greater values tend to overestimate transverse strains near the peak. The dilatancy parameter that, on average, best fits the experimental data is $\alpha_{p}=0.4$. In the second test, where passive confinement is provided, a value of $\alpha_{p}=0.1$ is needed to adjust the experimental results. Finally, in the third test, the value that is needed to capture the shear strength is $\alpha_{p}=0.6$. Low values of the dilatancy parameter, underestimate the shear strength.

One phenomenon that introduces disagreement between experimental and simulation is the presence of confinement. A confining pressure tends to 


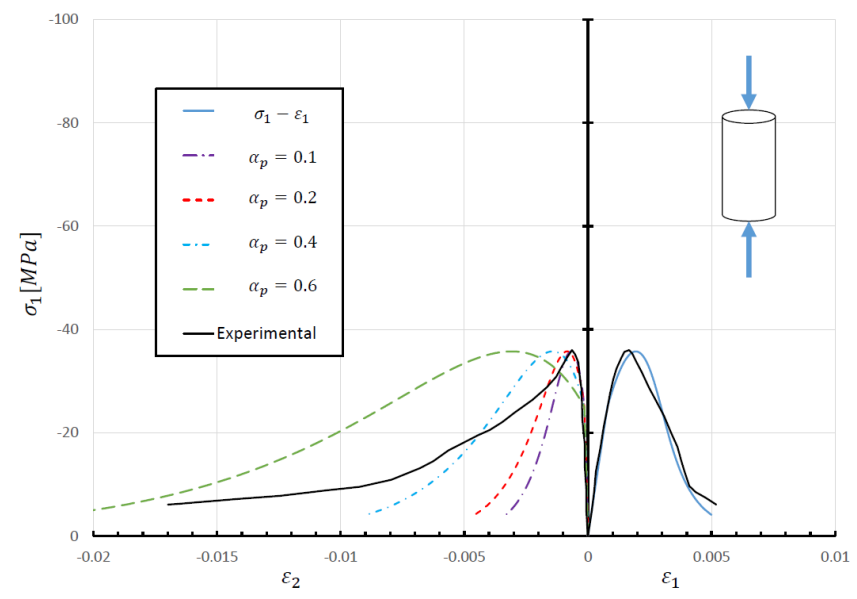

Figure 1: Longitudinal stress $\sigma_{1}$ vs longitudinal $\varepsilon_{1}$ and transverse $\varepsilon_{2}$ strains - Uniaxial Test. Experimental results by [20]

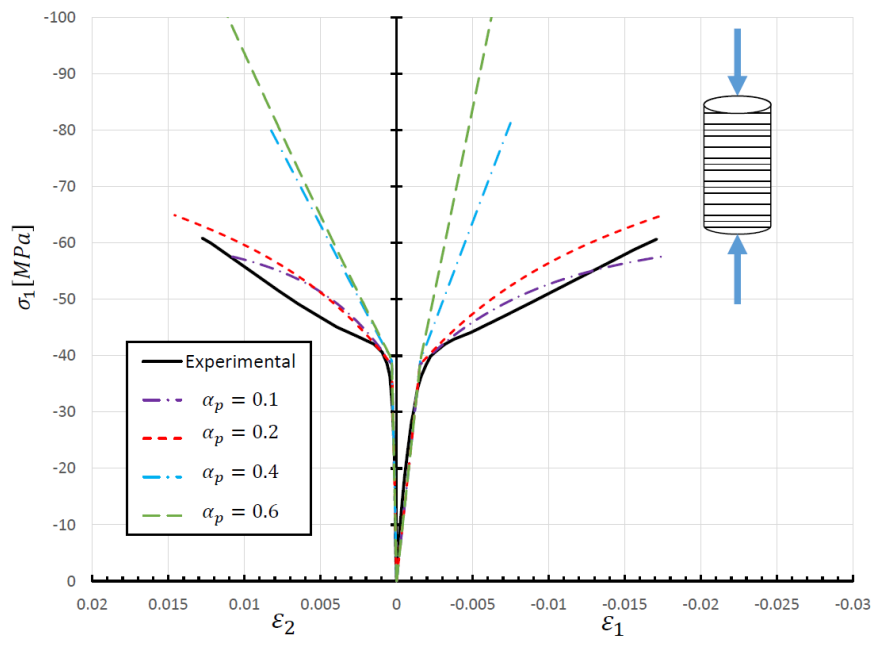

Figure 2: Longitudinal stress $\sigma_{1}$ vs longitudinal $\varepsilon_{1}$ and transverse $\varepsilon_{2}$ strains - Uniaxial Confined Test. Experimental results by [21]

reduce the effect of the dilatancy in concrete [20]. This makes the cases where passive confinement is present strongly dependent on the dilatancy parameter. Such dependence can be explained because small changes in the transverse expansion may cause variations on the confining pressure and consequently on the dilatancy. Another source of difference results from the evolution of damage. In the shear dominant cases, damage occurs in both 


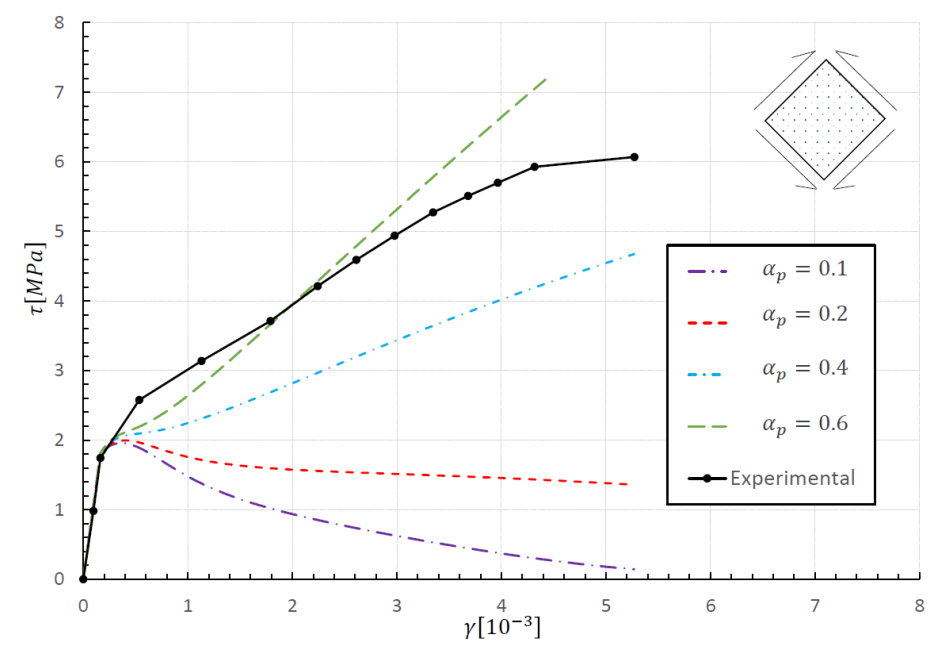

Figure 3: Shear stress $\tau$ vs shear strain $\gamma$ - Shear Panel Test. Experimental results by [22]

tensile and compressive directions. This produces a quick evolution of the concrete expansion and, consequently, large values of the dilatancy parameter are needed.

As commented above, in previous researches, ad hoc calibration of this parameter was needed for different loading cases. This leads to a loss of objectivity that needs to be reviewed. In addition, it can be seen that a constant parameter is not consistent with the experimental evidence.

\section{Proposed model}

The evolution of dilatancy in inelastic processes has been studied before in the field of soils and rock mechanics [23-26]. Nevertheless, when it comes to concrete, studies are scant. Some models exist focusing on the effect of variable volumetric expansion due to dilation $[8 ; 27 ; 28]$. However, to the knowledge of the authors, the role in the shear strength and other structural performances has not been investigated.

Vermeer and de Borst [29] proposed a dilatancy angle that depends on plastic strains. Oller et al. [19] introduced an explicit function of plasticdamage variables to control the evolution of the dilatancy angle. In the case of rock mechanics, Alejano and Alonso [23]; Rahjoo and Eberhardt [24]; Detournay [25]; Zhao and Cai [26] reported that the dilatancy parameter not only depends on the plastic state but also depends on the stress state, 
specially on confining stresses.

Based on the previous review and on the parametric analysis performed in section 2.4, it can be concluded that the dilatancy phenomena is affected by the confining pressure and the plastic-damage state. In consequence, the dilatancy angle is here proposed to be an explicit function of the plasticdamage and stress states. Taking into account Eq.(7), the $\alpha_{p}$ parameter can be written, in general terms, as follows, see Eq.(8).

$$
\alpha_{p}=\alpha_{p}(\boldsymbol{\kappa}, \overline{\boldsymbol{\sigma}})=\tan \psi(\boldsymbol{\kappa}, \overline{\boldsymbol{\sigma}})
$$

In the following, the evolution of the dilatancy angle proposed in this work is presented. First, the evolution of the dilatancy parameter with the plasticdamage state will be addressed independently of the stress state. Later, the effect of stresses on the dilatancy angle will be presented.

\subsection{Evolution with the plastic-damage state}

The plastic-damage state of the material is expressed through two variables, one for tension $\kappa_{t}$ and other for compression $\kappa_{c}$. Both are defined for uniaxial processes. In order to establish the evolution law, first, the definition of a plastic-damage variable for multiaxial states needs to be defined. In order to develop this task, the following physical aspects should to be taken into account.

The effect of dilatancy is greater and evolves quickly in those cases where the material is subjected to shear stresses, as in Fig.(3). Further, on an hypothetical case where the material is first damaged in tension and then subjected to compression, dilatancy would evolve quicker than in a pure compressive case. This is due to the development of microcracks in tension. When compression is later applied, the microcracks cannot be perfectly closed because the surface of the cracks had suffered small displacements. This increases the dilatancy and the volumetric expansion.

Bearing in mind the mentioned behavior, a scalar plastic-damage variable $k_{m}$ for multiaxial cases is proposed, see Eq.(9)

$$
\left(1-\kappa_{m}\right)=\left[1-\{1+\eta r(\overline{\boldsymbol{\sigma}})\} \kappa_{t}\right] \cdot\left[1-\{1-r(\overline{\boldsymbol{\sigma}})\} \kappa_{c}\right]
$$

where $\eta$ is a constant which value is set equal to 20 to give more importance to the tensile plastic-damage. This factor is proposed to fit experimental data. $r(\overline{\boldsymbol{\sigma}})$ is a weight factor that depends on the principal values of 
the effective stress tensor $\hat{\overline{\boldsymbol{\sigma}}}$. It is equal to zero for triaxial compression and equals to one for triaxial tension, as can be seen in Eq.(10).

$$
r(\hat{\overline{\boldsymbol{\sigma}}})=\frac{\sum_{i=1}^{3}\left\langle\hat{\bar{\sigma}}_{i}\right\rangle}{\sum_{i=1}^{3}\left|\hat{\bar{\sigma}}_{i}\right|}
$$

In triaxial compression, the variable defined in Eq.(9) is equal to $\kappa_{m}=$ $1-\left(1-\kappa_{t}\right)\left(1-\kappa_{c}\right)$. It can be seen that it takes into account the previous tensile plastic-damage.

The evolution of the dilatancy angle can be explained by considering concrete as a granular material. During inelastic processes, particles slide past each other on the surface of microcracks. This increases both the internal friction and the total volume. Thus, the evolution of the dilatancy angle $\psi$ is related to the evolution of the internal friction angle $\phi$. The following evolution for both angles is proposed as in [19], see Eqs.(11) and (12).

$$
\begin{gathered}
\psi=\left\{\begin{array}{cc}
0 & \text { if } \phi \leq \phi_{c v} \\
\arcsin \left(\frac{\sin \phi-\sin \phi_{c v}}{1-\sin \phi \sin \phi_{c v}}\right) & \text { if } \phi>\phi_{c v}
\end{array}\right. \\
\sin \phi=\left\{\begin{array}{cc}
\frac{2 \sqrt{\kappa_{m}}}{1+\kappa_{m}} \sin \phi^{\text {peak }} & \text { if } \kappa_{m} \leq 1 \\
\sin \phi^{\text {peak }} & \text { if } \kappa_{m}>1
\end{array}\right.
\end{gathered}
$$

where $\phi^{\text {peak }}$ and $\psi^{\text {peak }}$ are the values of the dilatancy and friction angles when the material is fully damaged. $\phi_{c v}$ is the internal friction angle at constant volume and is calculated as in Eq.(13). It can be interpreted as the value of the friction angle when dilatancy begins.

$$
\sin \phi_{c v}=\frac{\sin \phi^{\text {peak }}-\sin \psi^{\text {peak }}}{1-\sin \phi^{\text {peak }} \sin \psi^{\text {peak }}}
$$

The proposed evolution of the dilatancy parameter with the plasticdamage variable is plotted in Fig.(4).

In soils, dilatancy at early stages has a negative value, but this effect is not evidenced in concrete [29]. First, the dilatancy parameter remains equal 


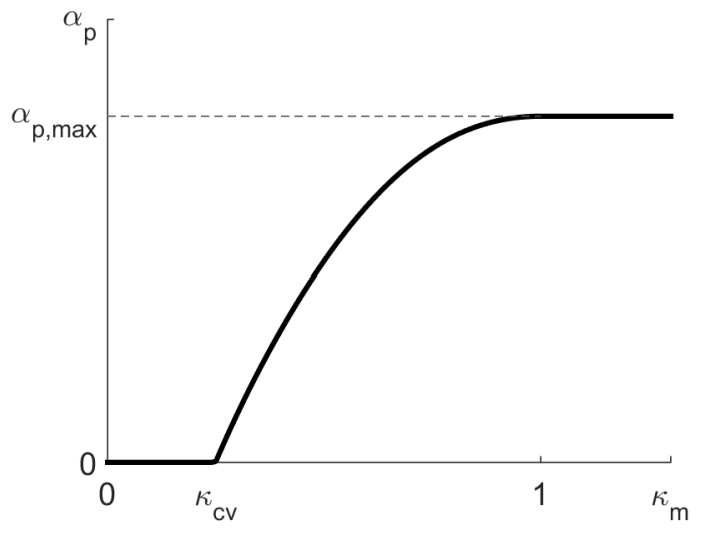

Figure 4: Dilatancy parameter vs damage

to zero until plastic-damage reaches the value of $\kappa_{c v}$. This is the value of the plastic-damage variable at constant volume when $\phi=\phi_{c v}$. Later, $\alpha_{p}$ increases up to its maximum value $\alpha_{p, \max }=\tan \psi^{\text {peak }}$ when the material is fully damaged $\kappa_{m}=1$.

Up to this point, the dilatancy parameter depends only on the plasticdamage state. The evolution is controlled by two parameters $\psi^{\text {peak }}$ and $\phi^{\text {peak }}$. In [19], they were considered as constant material properties. In the current research, it is proposed to vary $\psi^{\text {peak }}$ and $\phi^{p e a k}$ as a function of the stress state, to consider the effect of confinement.

\subsection{Influence of the stress state}

The experimental observations indicates that the presence of confinement reduces significantly the effect of dilatancy, as it was shown in section (2.4). Further, the maximum dilatancy is observed in the cases dominated by high shear stresses.

Therefore, in this research, it is proposed to affect the peak values of the internal friction and dilatancy angles by a term that depends on the stress state, see Eqs.(14) and (15).

$$
\begin{gathered}
\phi^{\text {peak }}=\phi^{\text {peak }}(\overline{\boldsymbol{\sigma}})=\phi^{\text {max }} e^{-a(1+b) \bar{I}_{1}} \\
\psi^{\text {peak }}=\psi^{\text {peak }}(\overline{\boldsymbol{\sigma}})=\psi^{\text {max }} e^{-a(1+b) \bar{I}_{1}}
\end{gathered}
$$




$$
a=\frac{1}{f_{c}} ; b=\frac{\overline{I_{1}}}{\sqrt{3 \bar{J}_{2}}}
$$

where $\phi^{\max }$ and $\psi^{\max }$ are material properties and are the maximum internal friction and dilatancy angles, respectively. $f_{c}$ is the uniaxial concrete strength. A similar term was proposed by [8] to modify the evolution of plastic-damage variables in presence of confinement. Here, it is used to modify the dilatancy and internal friction peak angles.

It can be seen that in the isotropic compression and tension cases, the exponent in Eq.(14) is not defined. However, in the compressive direction this is not relevant as the yield function is open in the hydrostatic axis. In the isotropic tensile point, the exponential term is taken equal to one to overcome this issue.

In Fig.(5) three curves are plotted for the evolution of the dilatancy parameter. Each one corresponds to a theoretical process for different degrees of constant confinement.

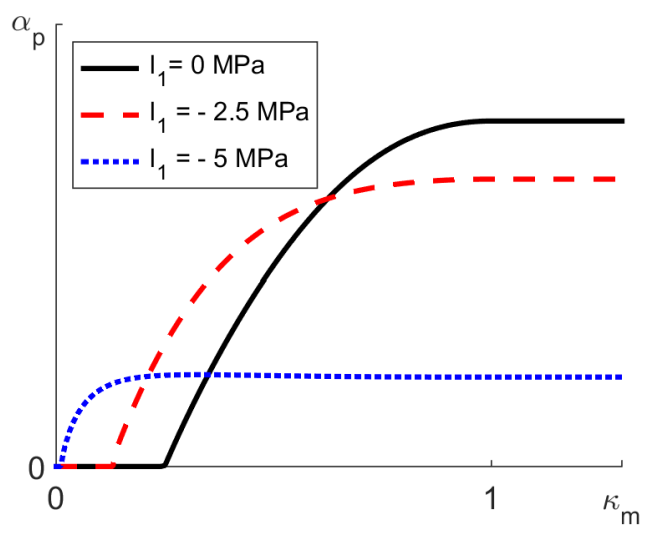

Figure 5: Dilatancy parameter vs damage for different confining pressures

As can be observed in Fig.(5), in the proposed model, the presence of a higher confinement produces a reduction on the peak value of the dilatancy parameter. Moreover, it produces an early onset of dilatancy.

\section{Validation}

The proposed model is validated by the simulation of three different sets of experimental data which are representative of very different failure modes. 
The first set consists in uniaxial compressive tests of concrete cylinders performed by Osorio et al. [20]. Four different concrete mixes were used from normal to high strength concrete. The transverse strains were measured in order to study the free expansion of the unconfined concrete.

The second experimental campaign, performed by Aire [21], involves six uniaxial compressive tests of concrete cylinders passively confined by different types of FRP jackets. Three different amounts of transverse reinforcements were tested using glass and carbon fibers. As FRP do not show yielding stress, modeling the confinement response is more sensitive to the adequate simulation of concrete expansion than in the case of steel confinement reinforcement.

Finally, fifteen reinforced concrete panels, tested by Vecchio and Collins [22], are simulated. The panels were subjected to in-plane shear, and each one had different amounts of reinforcement as well as different concrete strengths.

The material parameters in the three tests are obtained from available data. If a material parameter is not reported in the original publication then, the corresponding value is estimated using Eqs.(16) as recommended in $[7 ; 30]$.

$$
\begin{array}{rlr}
E & =\left(0.8+0.2 \frac{f_{c}+8}{88}\right) \sqrt[3]{f_{c}+8} & {[M P a]} \\
f_{t} & =0.302 f_{c}^{2 / 3} & {[M P a]} \\
G_{t} & =0.073 f_{c}^{0.18} & {[N / m m]} \\
G_{c} & =\left(\frac{f_{c}}{f_{t}}\right)^{2} G_{t} & \\
\nu & =0.2 &
\end{array}
$$

The calibration of the stiffness degradation response is made by means of the two following material parameters.

$$
\bar{D}_{c}=0.423\left(1-\frac{f_{c}}{\varepsilon_{0} E}\right) ; \bar{D}_{t}=0.5
$$

Where $\bar{D}_{c}$ is the value of the stiffness degradation at the maximum compressive stress $f_{c}$, and $\varepsilon_{0}$ is the peak strain. The constant on the first expression in Eq.(17) is obtained from cyclic compressive tests in [20]. The tensile 
counterpart $\bar{D}_{t}$, is defined as the stiffness degradation at a stress equals to the half of the maximum tensile stress.

The values of the maximum internal friction and dilatancy angles in Eq.(14) control the evolution of dilatancy. The following values are used in all the simulations.

$$
\phi^{\max }=65^{\circ} ; \psi^{\max }=32^{\circ}
$$

\subsection{Unconfined concrete}

In this section, four concrete mixes, with different values of strength, are numerically tested and compared against experimental data from [20]. The parameters, based on the materials properties models shown in the set of Eqs.(16) and (17) are determined from the compressive strength of each test : $f_{c}=35,45,60$ and $80 \mathrm{MPa}$. The characteristic length is set as $l_{c h}=175 \mathrm{~mm}$, which is obtained as the cubic root of the volume of the test sample.

Figure (6) presents experimental and numerical results for each one of the concrete mixes. Two numerical curves are traced, one obtained with the original model of $[5 ; 6]$ with a constant dilatancy parameter equal to $\alpha_{p}=0.2$, and the other calculated with the proposed model. In Fig.(6), $\varepsilon_{1}$ and $\sigma_{1}$ are the strain and stress in the load direction respectively, and $\varepsilon_{2}$ is the transverse strain.

It can be observed, in Fig.(6), that a fine agreement between numerical and experimental results is obtained with the proposed model. Particularly, it should be noticed that the lateral expansion, represented by $\varepsilon_{2}$, is well traced by the proposed model while the constant dilatancy control model underestimates the transverse strains. The main differences appear in the case of high strength concrete $\left(f_{c}=80 \mathrm{MPa}\right)$, where the experimental data presents a snap-back behavior after the peak load, which is not manifested in the simulation. However, considering the complexity of the control of the test, particularly in the case of high strength specimens as reported in [20], this difference is considered acceptable.

\subsection{Confined concrete with different confining materials}

The simulation of six passively confined concrete specimens is presented in this section. The transverse reinforcement consists of glass and carbon FRP jackets using 1, 3 or 6 plies of each material. Jackets are simulated in this validation by means of its transverse reinforcement ratios in the direction 


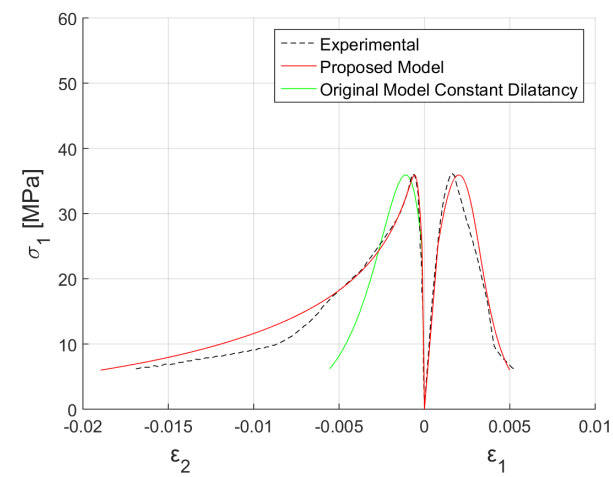

(a) H35

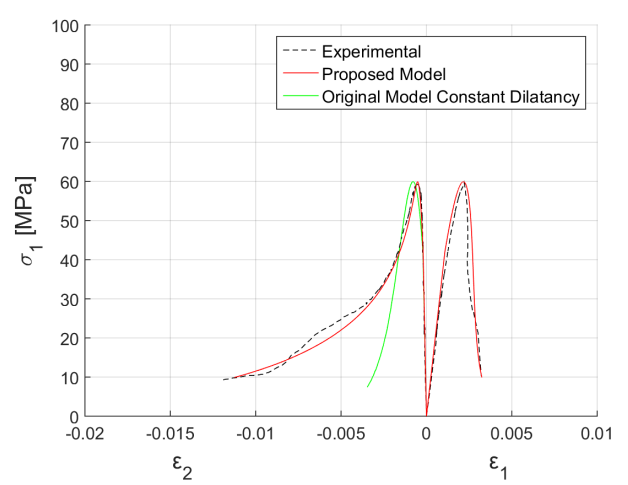

(c) H60

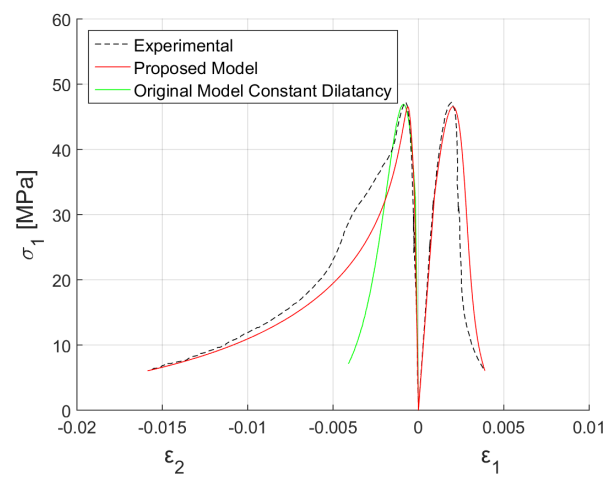

(b) H45

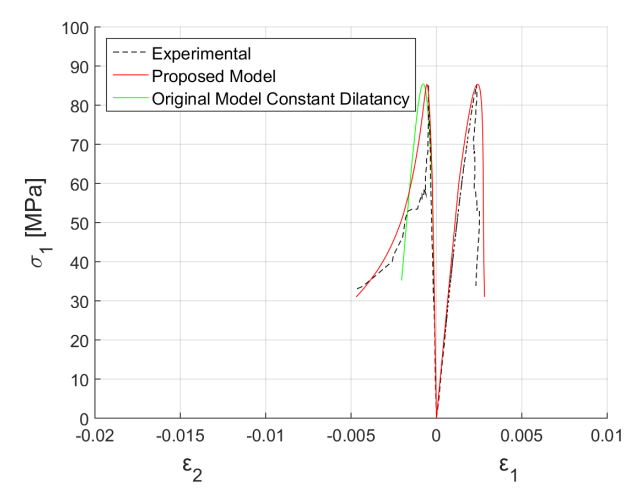

(d) H80

Figure 6: $\sigma_{1}-\varepsilon_{1}$ and $\sigma_{1}-\varepsilon_{2}$ curves for different concrete mixes. Experimental results by [20]

transverse to the load application, as can be seen in the equilibrium equation (19).

$$
\sigma_{t r}+\rho_{r} \sigma_{r}=0
$$

Where $\sigma_{t r}$ is the transverse stress in concrete, $\rho_{r}$ is the reinforcement ratio, which, in the case of jackets, is calculated as its thickness divided by the radius of the concrete specimen. $\sigma_{r}$ is the stress in the reinforcement. Perfect bond is assumed between the jacket and the concrete mass.

Material properties of the GFRP and CFRP reported in [21] are summarized in Table 1.

The concrete properties used in the six tests were determined from Eqs. 


\begin{tabular}{lccc} 
& $e[\mathrm{~mm}]$ & $E[\mathrm{GPa}]$ & $f_{u}[\mathrm{GPa}]$ \\
\hline Glass & 0.149 & 65 & 3.0 \\
Carbon & 0.117 & 240 & 3.9
\end{tabular}

Table 1: FRP properties of 1 ply

(16) and (17) using $f_{c}=42 M P a$ and $\varepsilon_{0}=0.00239$. The deformation modulus of concrete is taken as $E=25 \mathrm{GPa}$, as reported in [21]. The characteristic length for the CFRP specimens is $l_{c h}=20 \mathrm{~mm}$, while in the case of GFRP $l_{c h}=10 \mathrm{~mm}$ is used. Both values correspond to the base length of the strain gauges used to measure transverse strains in the physical test.

Fig.(7) compares the numerical and experimental results, obtained for each confinement material and for the different amounts of confining reinforcement.

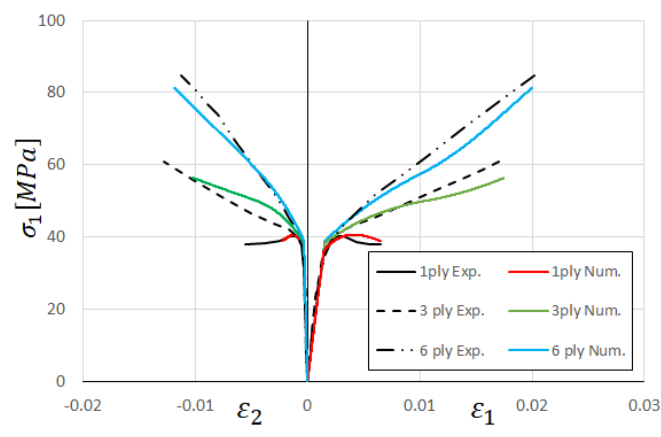

(a) GFRP

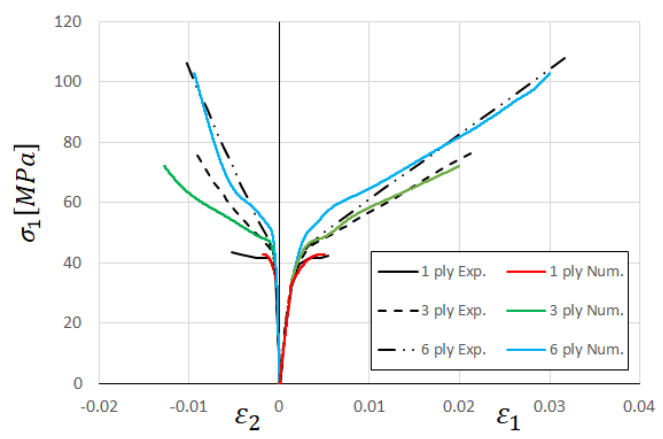

(b) CFRP

Figure 7: $\sigma_{1}-\varepsilon_{1}$ and $\sigma_{1}-\varepsilon_{2}$ curves for (a) Glass and (b) Carbon FRP jackets. Experimental results by $[21]$

It can be seen that the enhancement in both strength and ductility of the passively confined specimens is well captured. In the case of GFRP jackets, a 5-8 \% loss of strength is predicted by the model. The main differences appear in the case of CFRP with 3 layers of reinforcement, where transverse strain is overestimated. The confined strength is adequately captured in all the cases. 


\subsection{Shear tests}

In this section, fifteen reinforced concrete panels subjected to pure inplane shear load, with different amounts of reinforcement and concrete strengths, are numerically reproduced. These panels belong to a larger experimental campaign [22] that included specimens under combined axial and shear loads. Here, only the pure shear tests are considered to study the effect of dilatancy in the predicted shear capacity of the proposed model.

The longitudinal and transverse reinforcement are included by means of the corresponding reinforcement ratios, similarly as in Eq.(19). The transverse reinforcement ratio is considered as elastic-perfectly plastic and perfectly bonded to the concrete mass. Ratios and yield stress of the reinforcement steel as well as the concrete properties are summarized in Table 2.

\begin{tabular}{ccccccc} 
Panel & $f_{c}[\mathrm{MPa}]$ & $\varepsilon_{0}$ & $f_{y l}[\mathrm{MPa}]$ & $\rho_{l}$ & $f_{y t}[\mathrm{MPa}]$ & $\rho_{t}$ \\
\hline PV3 & 26.6 & 0.0023 & 662 & 0.00483 & 662 & 0.00483 \\
PV4 & 26.6 & 0.0025 & 242 & 0.01056 & 242 & 0.01056 \\
PV6 & 29.8 & 0.0025 & 266 & 0.01785 & 266 & 0.01785 \\
PV9 & 11.6 & 0.0028 & 455 & 0.01785 & 455 & 0.01785 \\
PV10 & 14.5 & 0.0027 & 276 & 0.01785 & 276 & 0.00999 \\
PV11 & 15.6 & 0.0026 & 235 & 0.01785 & 235 & 0.01306 \\
PV12 & 16 & 0.0025 & 469 & 0.01785 & 269 & 0.00446 \\
PV13 & 18.2 & 0.0027 & 248 & 0.01785 & - & 0 \\
PV16 & 21.7 & 0.002 & 255 & 0.0074 & 255 & 0.0074 \\
PV18 & 19.5 & 0.0022 & 431 & 0.01785 & 412 & 0.00315 \\
PV19 & 19 & 0.00215 & 458 & 0.01785 & 299 & 0.00713 \\
PV20 & 19.6 & 0.0018 & 460 & 0.01785 & 297 & 0.00885 \\
PV21 & 19.5 & 0.0018 & 458 & 0.01785 & 302 & 0.01296 \\
PV22 & 19.6 & 0.002 & 458 & 0.01785 & 420 & 0.01524 \\
PV27 & 20.5 & 0.0019 & 442 & 0.01785 & 442 & 0.01785
\end{tabular}

Table 2: Panels reinforcement ratios and material properties

The material parameters are calculated using Eqs.(16) and (17). In panels PV3, 4, 6, 9, 11 and 16, the tensile strength is determined using the following expression suggested in the original publication [22]

$$
f_{t}=0.33 \sqrt{f_{c}}
$$


The characteristic length used in the tests is $l_{c h}=140 \mathrm{~mm}$, which corresponds to the cubic root of the measured volume.

Figures (8) to (12) present the results of panels PV4, 13, 16, 18 and 22. The results for the rest of the panels are reported as supplementary material to this paper. For each panel, three curves are shown in each figure: the shear stress-strain $\tau-\gamma$, the normalized principal compressive stress-strain $\sigma_{d} / f_{c}-\varepsilon_{d} / \varepsilon_{0}$ and the normalized principal tensile stress-strain $\sigma_{d t} / f_{t}-\varepsilon_{d t}$. Numerical results are obtained with the proposed model and with the original model by $[5 ; 6]$ with a constant dilatancy parameter equal to $\alpha_{p}=0.2$.

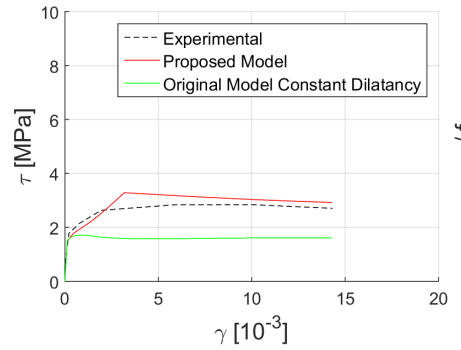

(a) Shear

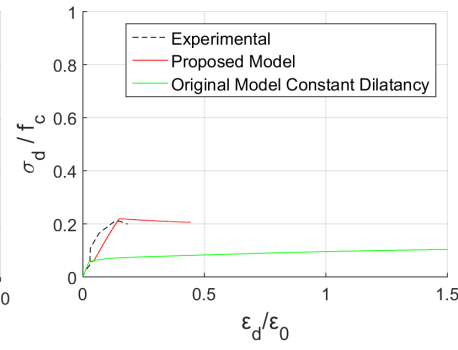

(b) Principal compression

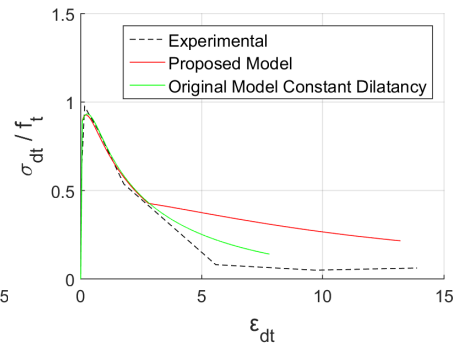

(c) Principal tension

Figure 8: Stress-strain plots of panel PV4. Experimental results by [22]

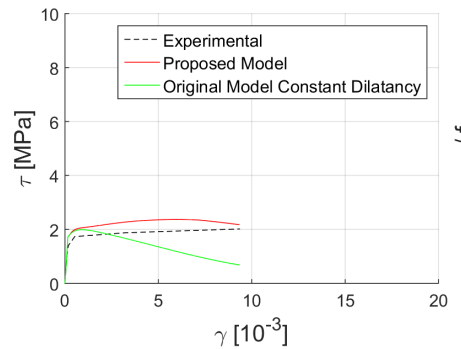

(a) Shear

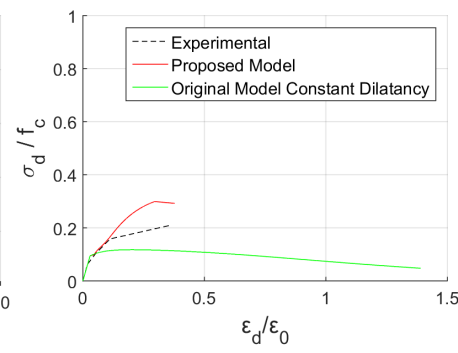

(b) Principal compression

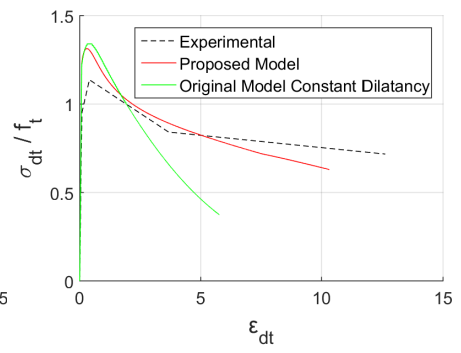

(c) Principal tension

Figure 9: Stress-strain plots of panel PV13. Experimental results by [22]

As can be seen in Figs.(8-12), the shear response of the panels is well estimated by the present model. An improvement with regard to the constant dilatancy model is appreciated as the original constant dilantancy model tends to predict larger material softening and less shear capacity, in general. 


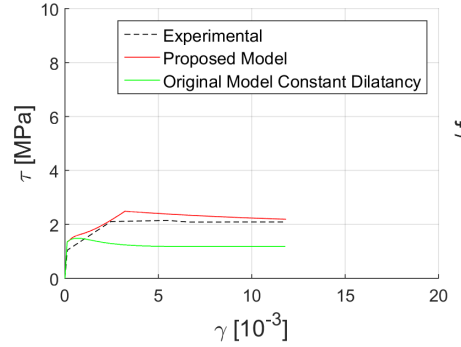

(a) Shear

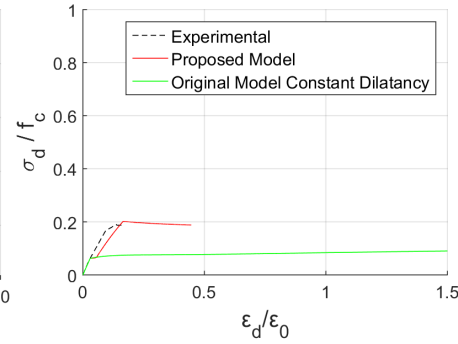

(b) Principal compression

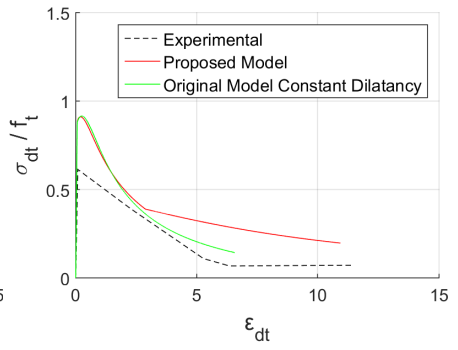

(c) Principal tension

Figure 10: Stress-strain plots of panel PV16. Experimental results by [22]

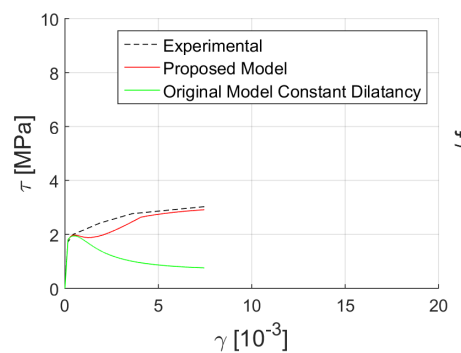

(a) Shear

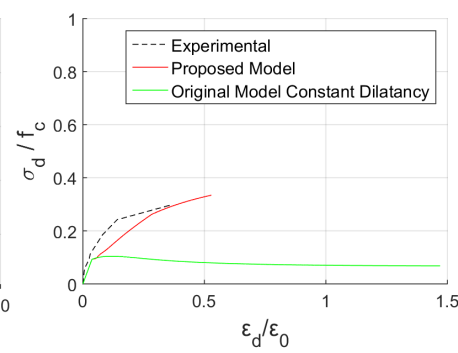

(b) Principal compression

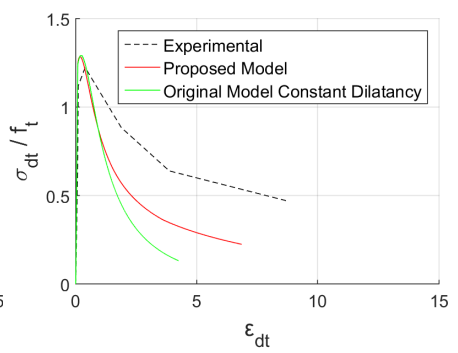

(c) Principal tension

Figure 11: Stress-strain plots of panel PV18. Experimental results by [22]

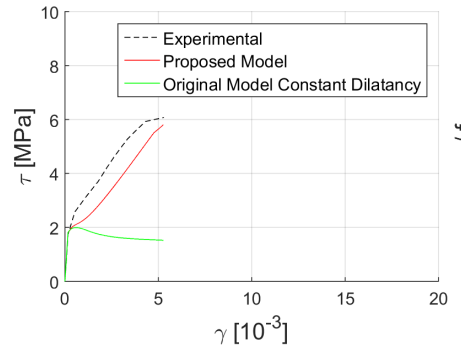

(a) Shear

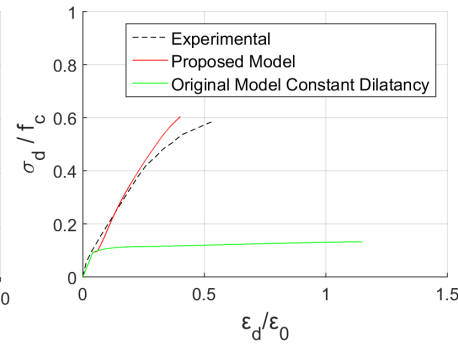

(b) Principal compression

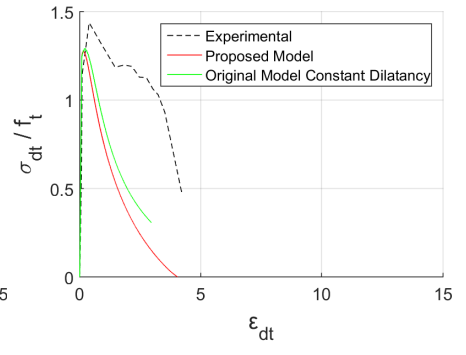

(c) Principal tension

Figure 12: Stress-strain plots of panel PV22. Experimental results by [22]

The studied panels exhibit different failure modes; including specimens showing yielding of both longitudinal and transverse reinforcement, yielding of transverse reinforcement prior to concrete failure and concrete failure without yielding of the reinforcements. The model shows to be able to capture 
different modes of failure adequately without a recalibration of the dilatancy or the fracture energy parameters.

\section{Conclusions}

A constitutive plastic-damage model for concrete with evolutive dilatancy is proposed. The model is based on the original model of Lee and Fenves [5, 6] which in turns relies on the model of [1]. The original model has a constant dilatancy parameter. In this paper, it is demonstrated that a constant value of the dilatancy parameter is not adequate to accurately predict the free expansion of concrete. Consequently, the original model has difficulties to trace the response under passive confinement or shear stresses.

A new constitutive plastic-damage model is here developed incorporating the variability of the dilatancy and friction angle parameters as explicit functions of the plastic-damage and stress states. This function produces the maximum dilatancy for uniaxial compression and pure shear states. The resulting dilatancy is automatically reduced when the confinement stresses increase.

The evolution of the dilatancy is controlled by two material properties, the maximum dilatancy and internal friction angles. These properties may be obtained through tests where different confinement stresses are considered. In this paper, values of $32^{\circ}$ for the maximum dilatancy angle and $65^{\circ}$ for the maximum inner friction angle are proposed. These values are used in the validation of the proposed model obtaining good agreement with experimental results.

The validation of the proposed model is carried out by simulating several experimental campaigns producing different modes of failure and phenomenological responses of concrete. Ordinary to high strength concrete samples were considered along the validation tests, ranging from 11 to 80 MPa.

The model shows to be capable of accurately trace the volumetric expansion of concrete in uniaxial compressive tests including softening and confinement. It is also shown to be capable of capturing the enhancement in strength and ductility when passive confinement is applied by means of different confining materials. Good estimation of concrete shear strength and softening behavior is obtained. The model response is objective on different modes of failure with the same material parameters. 
The adequate control of the dilatant behavior of concrete is shown to be of paramount importance as it controls the volumetric expansion and, consequently, affects the strength and ductility of confined concrete as well as the shear strength and softening.

The proposed dilatancy model contributes to extend the capabilities of the plastic-damage model in the simulation of reinforced concrete elements and structures in a consistent manner for different types of load and failure modes.

\section{Acknowledgments}

The authors acknowledge the support provided by Spanish Ministry of Economy, Industry and Competitiveness and the European Regional Development Fund (ERDF), through the projects BIA2015-64672-C4-1R and BIA2012-36848. The first author acknowledges the support of the Catalan Agency for University and Research through doctoral scholarship 2018FI-B100103 .

\section{References}

[1] J. Lubliner, J. Oliver, S. Oller, E. Oñate, A plastic-damage model for concrete, International Journal of Solids and Structures 25 (1989) 299 -326 .

[2] J. Coleman, E. Spacone, Localization issues in force-based frame elements, Journal of Structural Engineering 127 (2001) 1257-1265.

[3] Z. P. Bažant, B. H. Oh, Crack band theory for fracture of concrete, Matériaux et Construction 16 (1983) 155-177.

[4] J. Oliver, A consistent characteristic length for smeared cracking models, International Journal for Numerical Methods in Engineering 28 (1989) 461-474.

[5] J. Lee, G. L. Fenves, Plastic-damage model for cyclic loading of concrete structures, Journal of Engineering Mechanics 124 (1998) 892-900.

[6] J. Lee, G. L. Fenves, A return-mapping algorithm for plastic-damage models: 3-D and plane stress formulation, International Journal for Numerical Methods in Engineering 50 (2001) 487-506. 
[7] B. Alfarah, F. López-Almansa, S. Oller, New methodology for calculating damage variables evolution in plastic damage model for RC structures, Engineering Structures 132 (2017) $70-86$.

[8] M. Moharrami, I. Koutromanos, Triaxial constitutive model for concrete under cyclic loading, Journal of Structural Engineering 142 (2016) 04016039 .

[9] ABAQUS, Theory manual version 6.12, Abaqus Documentation (2012).

[10] B. Luccioni, S. Oller, R. Danesi, Coupled plastic-damaged model, Computer Methods in Applied Mechanics and Engineering 129 (1996) 81 89.

[11] B. Luccioni, V. Rougier, A plastic damage approach for confined concrete, Computers \& Structures 83 (2005) 2238 - 2256.

[12] J. Y. Wu, J. Li, R. Faria, An energy release rate-based plastic-damage model for concrete, International Journal of Solids and Structures 43 (2006) $583-612$.

[13] A. Saritas, F. C. Filippou, Numerical integration of a class of 3D plasticdamage concrete models and condensation of 3D stress-strain relations for use in beam finite elements, Engineering Structures 31 (2009) 2327 -2336 .

[14] A. S. Genikomsou, M. A. Polak, Finite element analysis of punching shear of concrete slabs using damaged plasticity model in Abaqus, Engineering Structures 98 (2015) $38-48$.

[15] A. Earij, G. Alfano, K. Cashell, X. Zhou, Nonlinear three-dimensional finite-element modelling of reinforced-concrete beams: Computational challenges and experimental validation, Engineering Failure Analysis 82 (2017) $92-115$.

[16] J. Nzabonimpa, W.-K. Hong, J. Kim, Nonlinear finite element model for the novel mechanical beam-column joints of precast concrete-based frames, Computers \& Structures 189 (2017) $31-48$.

[17] J. Lemaitre, J.-L. Chaboche, Mechanics of Solid Materials, Cambridge University Press, 1990. 
[18] O. Omidi, V. a. Lotfi, Finite element analysis of concrete structures using plasticdamage model in 3-D implementation, International Journal of Civil Engineering 8 (2010).

[19] S. Oller, J. Oliver, J. Lubliner, E. Oñate, Un modelo constitutivo de daño plástico para materiales friccionales. parte i: Variables fundamentales, funciones de fluencia y potencial, Revista Internacional de Métodos Numéricos para Cálculo y Diseño en Ingeniería 4 (1988) 397431.

[20] E. Osorio, J. M. Bairán, A. R. Marí, Lateral behavior of concrete under uniaxial compressive cyclic loading, Materials and Structures 46 (2013) 709-724.

[21] C. Aire, Estudio experimental del comportamiento del hormigón confinado sometido a compresión, Ph.D. thesis, Departament D'Enginyeria de la Construcció, Universitat Politècnica de Catalunya, Barcelona, 2002 .

[22] F. Vecchio, M. Collins, The response of reinforced concrete to in-plane shear and normal stresses, Technical Report 82-03, Department of Civil Engineering, University of Toronto, 1982.

[23] L. Alejano, E. Alonso, Considerations of the dilatancy angle in rocks and rock masses, International Journal of Rock Mechanics and Mining Sciences 42 (2005) 481 - 507.

[24] M. Rahjoo, E. Eberhardt, A simplified dilation model for modeling the inelastic behavior of rock, in: ARMA 50th US Rock Mechanics/Geomechanics Symposium, American Rock Mechanics Association, 2016.

[25] E. Detournay, Elastoplastic model of a deep tunnel for a rock with variable dilatancy, Rock Mechanics and Rock Engineering 19 (1986) 99-108.

[26] X. Zhao, M. Cai, A mobilized dilation angle model for rocks, International Journal of Rock Mechanics and Mining Sciences 47 (2010) 368 384 . 
[27] S. J. Pantazopoulou, R. H. Mills, Microstructural aspects of the mechanical response of plain concrete, ACI Materials Journal 92 (1995).

[28] S. J. Pantazopoulou, Role of expansion on mechanical behavior of concrete, Journal of Structural Engineering 121 (1995) 1795-1805.

[29] P. Vermeer, R. de Borst, Non associated plasticity for soils, concrete and rock, Heron 29 (1983) 1-64.

[30] CEB-FIB, Model Code 2010, Thomas Telford, London, 2010. 


\title{
A new concrete plastic-damage model with an evolutive dilatancy parameter
}

\author{
Mauro Poliotti, Jesús-Miguel Bairán \\ Department of Civil and Environmental Engineering, Technical University of Catalonia, \\ (UPC), Jordi Girona, 1-3, Campus Nord, Mod. C-1, 08034 Barcelona, Spain
}

\section{Supplementary Material}

This file contains the numerical and experimental results for panels PV3, PV6, PV9, PV10, PV11, PV12, PV19, PV20, PV21 and PV27. Numerical results are obtained withe the proposed model with evolutive dilatancy. Three curves are presented for each panel: the shear stress-strain $\tau-\gamma$, the normalized principal compressive stress-strain $\sigma_{d} / f_{c}-\varepsilon_{d} / \varepsilon_{0}$ and the normalized principal tensile stress-strain $\sigma_{d t} / f_{t}-\varepsilon_{d t}$.

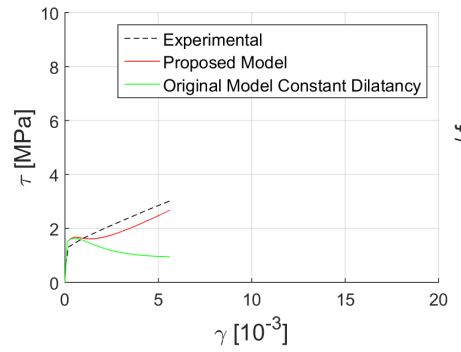

(a) Shear

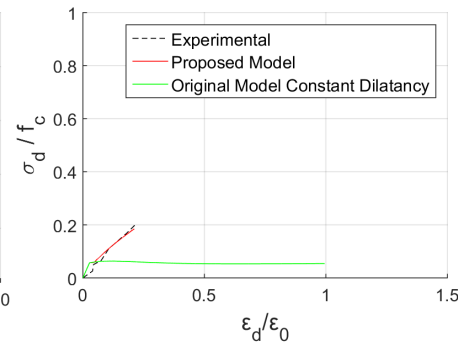

(b) Principal compression

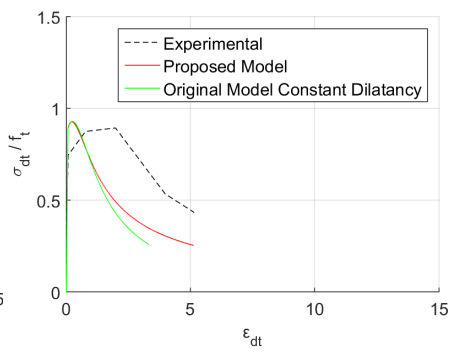

(c) Principal tension

Figure 1: Stress-strain plots of panel PV3. Experimental results by [22]

Email addresses: mauro.poliotti@upc.edu (Mauro Poliotti), jesus.miguel.bairan@upc .edu (Jesús-Miguel Bairán)

1

Please cite this paper as follows:

Poliotti M, Bairan JM, "A new concrete plastic-damage model with an evolutive dilatancy parameter", Engineering Structures, 2019, doi.org/10.1016/j.engstruct.2019.03.086. 


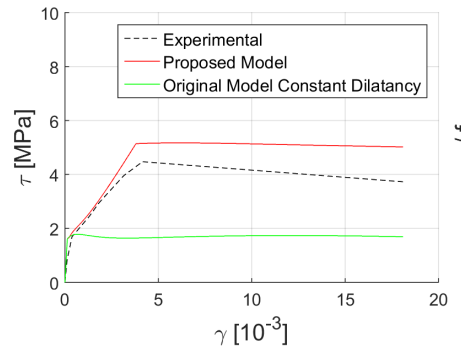

(a) Shear

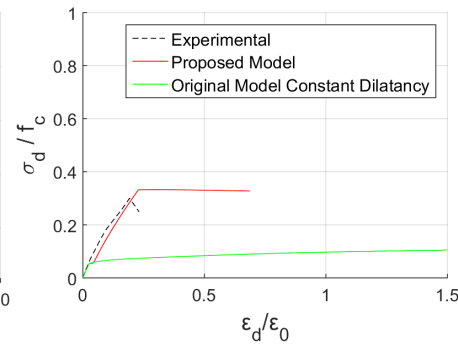

(b) Principal compression

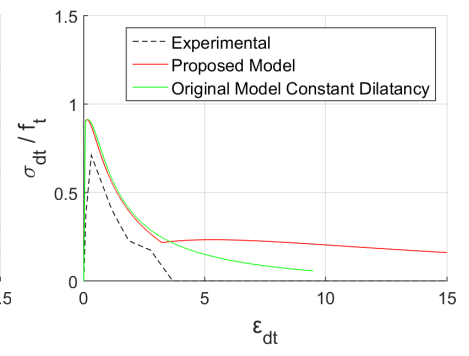

(c) Principal tension

Figure 2: Stress-strain plots of panel PV6. Experimental results by [22]

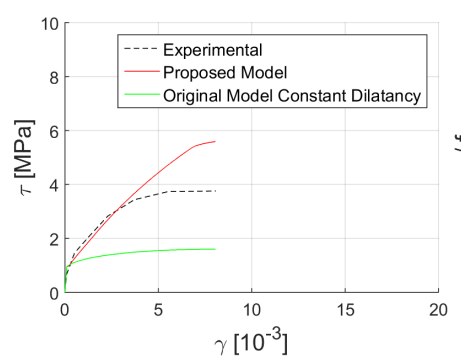

(a) Shear

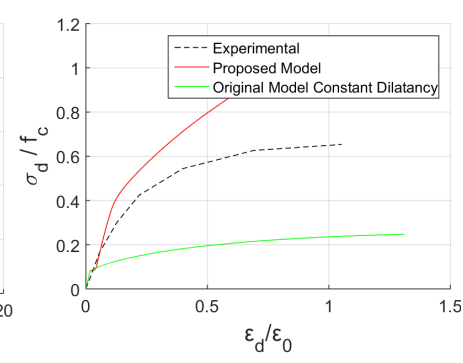

(b) Principal compression

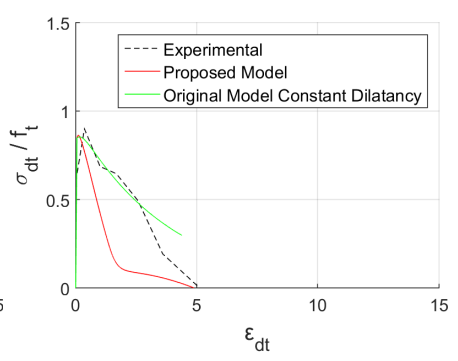

(c) Principal tension

Figure 3: Stress-strain plots of panel PV9. Experimental results by [22]

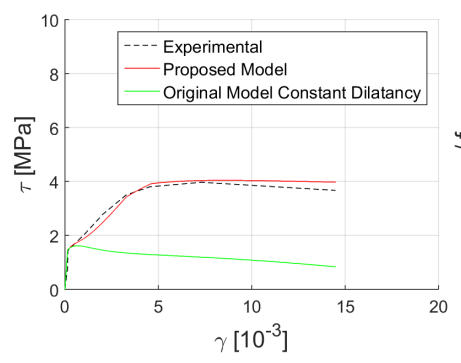

(a) Shear

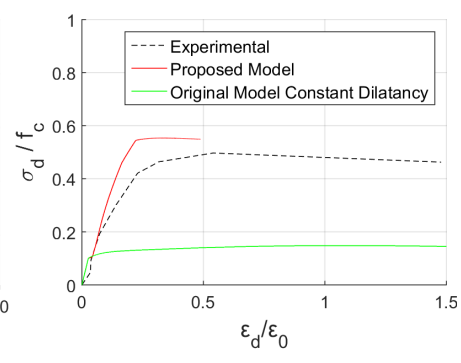

(b) Principal compression

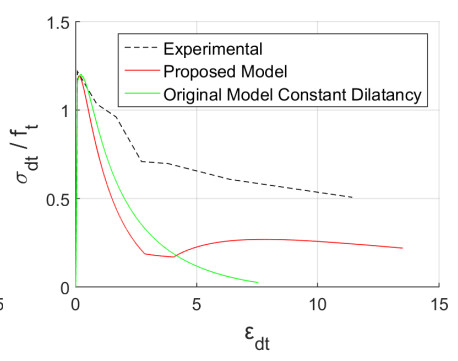

(c) Principal tension

Figure 4: Stress-strain plots of panel PV10. Experimental results by [22] 


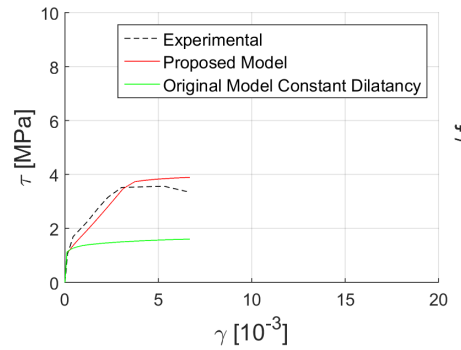

(a) Shear

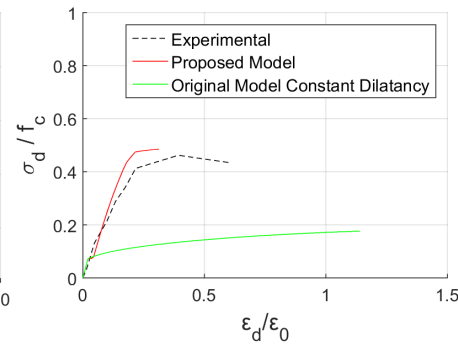

(b) Principal compression

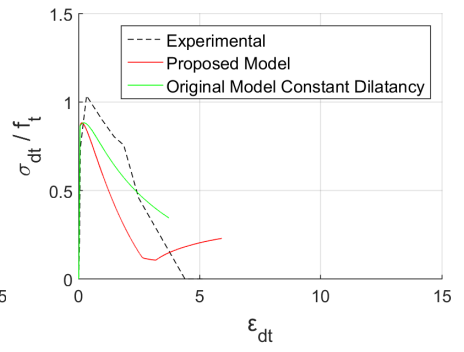

(c) Principal tension

Figure 5: Stress-strain plots of panel PV11. Experimental results by [22]

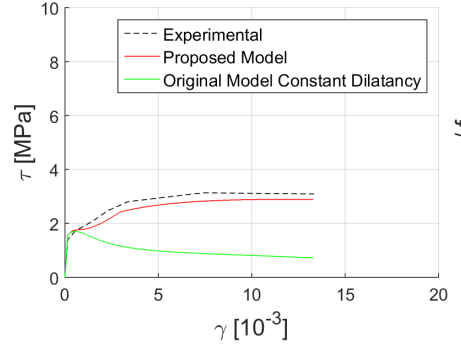

(a) Shear

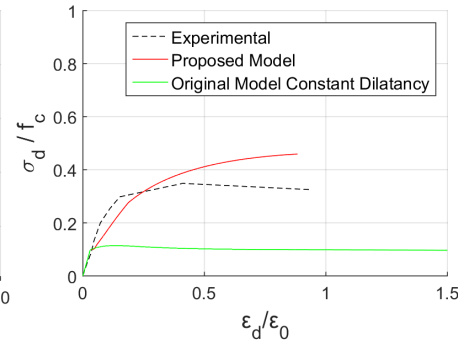

(b) Principal compression

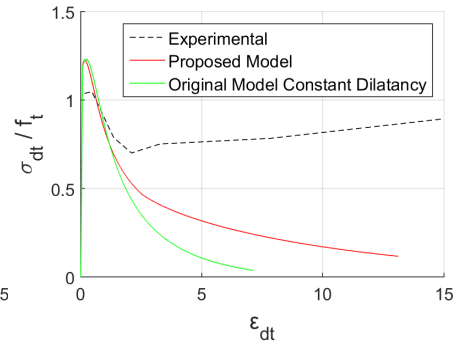

(c) Principal tension

Figure 6: Stress-strain plots of panel PV12. Experimental results by [22]

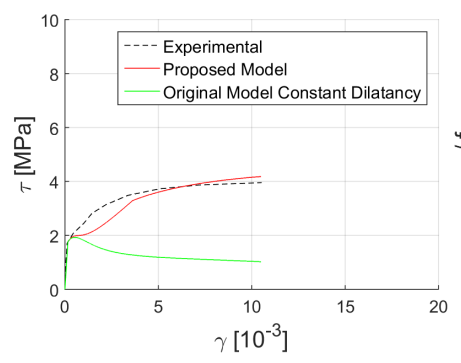

(a) Shear

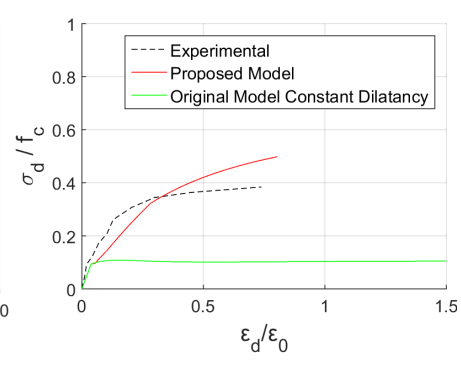

(b) Principal compression

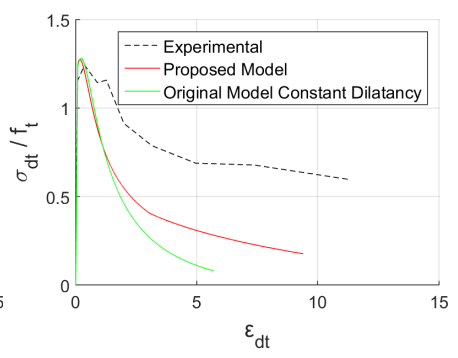

(c) Principal tension

Figure 7: Stress-strain plots of panel PV19. Experimental results by [22] 


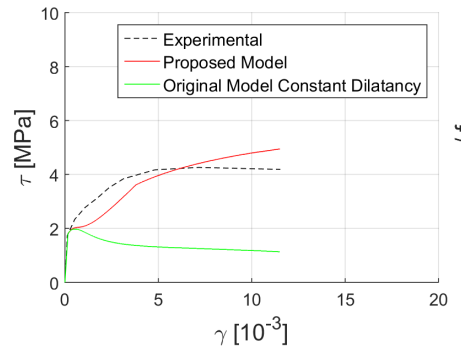

(a) Shear

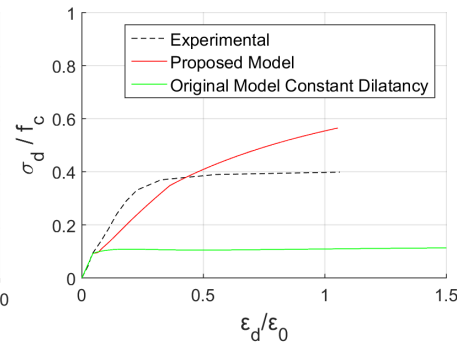

(b) Principal compression

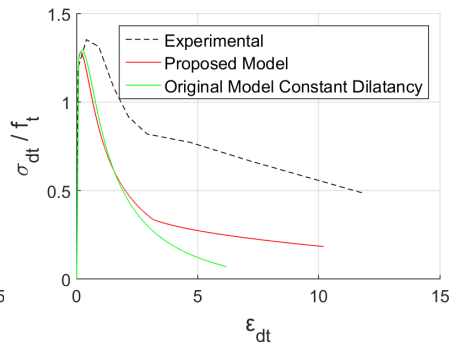

(c) Principal tension

Figure 8: Stress-strain plots of panel PV20. Experimental results by [22]

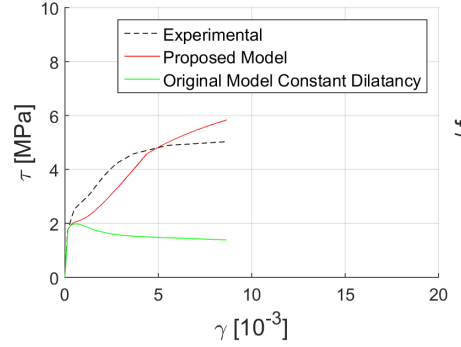

(a) Shear

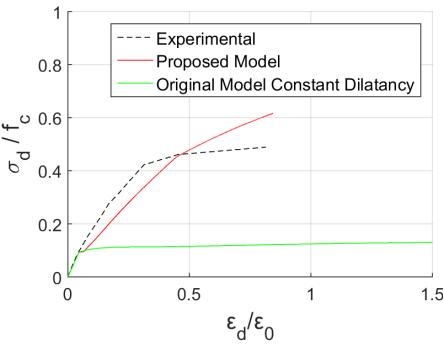

(b) Principal compression

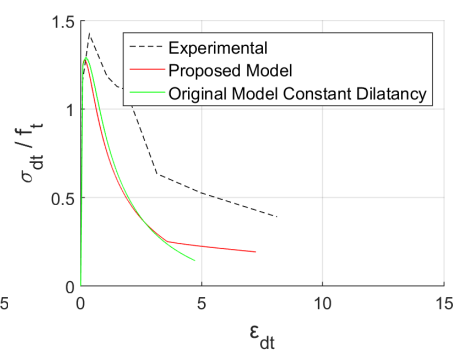

(c) Principal tension

Figure 9: Stress-strain plots of panel PV21. Experimental results by [22]

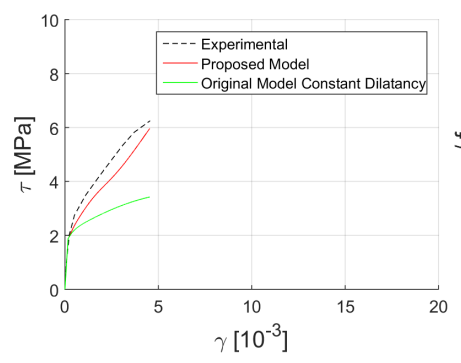

(a) Shear

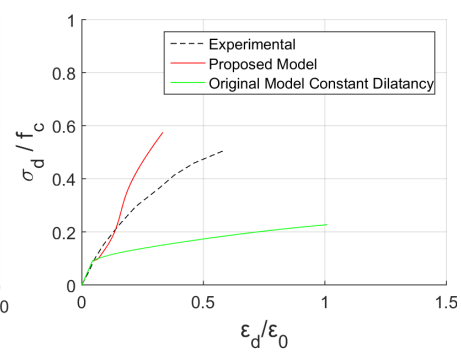

(b) Principal compression

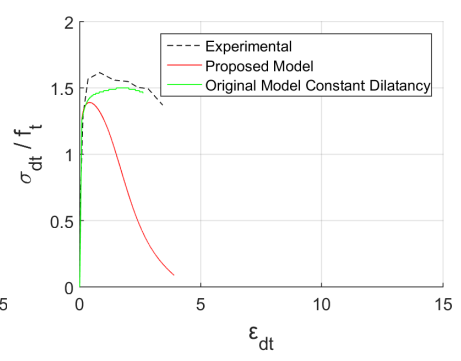

(c) Principal tension

Figure 10: Stress-strain plots of panel PV27. Experimental results by [22] 